\title{
A INIBIÇÃO NA SÍNTESE DE GIBERELINA REDUZ O CRESCIMENTO VEGETATIVO EM MACIEIRAS E PROPORCIONA CONTROLE DE “BITTER PIT” NOS FRUTOS'
}

\author{
JOÃO PAULO GENEROSO SILVEIRA², CASSANDRO VIDAL TALAMINI DO AMARANTE ${ }^{3}$ \\ CRISTIANO ANDRÉ STEFFENS ${ }^{4}$, AQUIDAUANA MIQUELOTO $^{2}$, \\ JOSÉ MASANORI KATSURAYAMA ${ }^{5}$
}

RESUMO - O "bitter pit" é um distúrbio fisiológico ocasionado pela deficiência de cálcio (Ca) em maçãs. No entanto, trabalhos recentes mostram que o "bitter pit" pode estar relacionado com aumento na atividade de giberelinas nas plantas. Este trabalho teve como objetivo avaliar os efeitos da pulverização de macieiras com um inibidor da síntese de giberelinas, o prohexadiona-cálcio (ProCa), e com giberelina $\mathrm{GA}_{3}$, no crescimento vegetativo das plantas e na ocorrência de "bitter pit". O experimento foi conduzido em um pomar localizado no município de São Joaquim-SC, na safra de 2009/2010. Macieiras 'Catarina' e 'Fuji' foram pulverizadas com água (tratamento-controle), ProCa e GA (ambos os produtos na dose de $319 \mathrm{mg} \mathrm{L}^{-1}$ ), na queda das pétalas (15-10-2009), quando as brotações do ano estavam com 5-10 cm de comprimento, sendo repetidas após 20 dias. Foram feitas avaliações foliares (teor de clorofila, área, massa seca e área específica), em janeiro/2010, e de comprimento dos ramos do ano e de peso dos ramos podados, em maio/2010. Os frutos foram colhidos na maturação comercial, armazenados em câmara fria convencional por quatro meses $\left(0 \pm 0,5^{\circ} \mathrm{C} / 90-95 \%\right.$ UR), e então avaliados quanto à ocorrência de "bitter pit" após cinco dias de vida de prateleira. Em ambas as cultivares, o crescimento vegetativo foi significativamente menor nas plantas tratadas com ProCa, e maior naquelas tratadas com $\mathrm{GA}_{3}$, comparativamente ao controle. Maçãs 'Catarina' e 'Fuji' do tratamento com ProCa apresentaram menor ocorrência de "bitter pit" após o período de armazenamento, associada aos menores teores de $\mathrm{K}, \mathrm{Mg}$ e N, em relação aos teores de $\mathrm{Ca}$, no tecido da casca, comparativamente ao tratamento-controle. Já o tratamento com $\mathrm{GA}_{3}$ aumentou a ocorrência de "bitter pit" em relação ao controle em ambas as cultivares.

Termos de indexação: Malus domestica Borkh, prohexadiona-cálcio, antigiberelina, fruto, composição mineral, pós-colheita, distúrbio fisiológico.

\section{INHIBITION OF GIBBERELLIN SYNTHESIS REDUCES VEGETATIVE GROWTH OF APPLE TREES AND PROVIDES CONTROL OF BITTER PIT IN THE FRUIT}

\begin{abstract}
Bitter pit is a physiological disorder caused by calcium $(\mathrm{Ca})$ deficiency in apples. However, recent publications have shown that bitter pit can be caused by an increase of gibberellins activity in the plant. This research was carried out to assess the effects of orchard spraying with prohexadione-calcium (ProCa) (an inhibitor of gibberellins synthesis) and gibberellin $\mathrm{GA}_{3}$ on vegetative growth of the trees and occurrence of bitter pit in the fruit. The experiment was conducted in an orchard located in São Joaquim, State of Santa Catarina (Southern Brazil), in 2009/2010. 'Catarina' and 'Fuji' apple trees were sprayed with water (control), ProCa and $\mathrm{GA}_{3}$ (both products at the dose of $319 \mathrm{mg} \mathrm{L}^{-1}$ ), at the petal fall stage (October 15, 2009), when shoots were 5-10 cm long, with treatments repeated after 20 days. The trees were evaluated in terms of chlorophyll content, area, dry mass and specific area of the leaves (in January/2010), and length of current season shoots and weight of shoots removed by winter pruning (in May/2010). Fruit were harvest at commercial maturity, cold stored $\left(0 \pm 0.5^{\circ} \mathrm{C} / 90-95 \% \mathrm{RH}\right)$ for four months, and then assessed for occurrence of bitter pit after five days of shelf life. In both cultivars, vegetative growth of the trees was reduced by ProCa and increased by $\mathrm{GA}_{3}$ in comparison to the control. 'Catarina' and 'Fuji' apples from trees treated with ProCa had lower occurrence of bitter pit after cold storage than the control treatment, as a result of lower contents of $\mathrm{K}, \mathrm{Mg}$ and $\mathrm{N}$, in relation to the Ca contents, assessed in the peel tissue of the fruit. On the other hand, the treatment with $\mathrm{GA}_{3}$ increased the occurrence of bitter pit compared to the control in both cultivars. Index terms: Malus domestica Borkh, prohexadione-calcium, anti-gibberellin, fruit, mineral composition, postharvest, physiological disorder.
\end{abstract}

\footnotetext{
'(Trabalho 188-11). Recebido em: 27-06-2011. Aceito para publicação em: 29-03-2012.

${ }^{2}$ Mestrandos em Produção Vegetal, Centro de Ciências Agroveterinárias0CAV/UDESC. Av. Luiz de Camões, 2090, CEP 88520-000, Lages-SC. E-mail: joaop-silveira@hotmail.com (autor para correspondência); aquidauanamiqueloto1@yahoo.com.br.

${ }^{3}$ Ph.D., Bolsista de Produtividade em Pesquisa do CNPq, Prof. do Depto. de Agronomia, CAV/UDESC. Av. Luiz de Camões, 2090 CEP 88520-000, Lages, SC. E-mail: amarante@cav.udesc.br

${ }^{4}$ Dr., Prof.do Dep ${ }^{\text {to }}$. de Agronomia, CAV/UDESC. Av. Luiz de Camões, 2090, CEP 88520-000, Lages-SC. E-mail: steffens@cav.udesc.br ${ }_{5}^{5}$ Pesq. da Epagri, Estação Experimental de São Joaquim. Cx. Postal 81, CEP 88600-000, São Joaquim-SC. E-mail: masanori@epagri.sc.gov.br
} 


\section{INTRODUÇÃO}

A cultura da macieira é de grande importância econômica para a região Sul do Brasil. No entanto, os produtores de maçã deparam-se com diversos problemas, principalmente relacionados às perdas pós-colheita ocasionadas por distúrbios fisiológicos. O "bitter pit" é um dos principais distúrbios, que pode ocasionar perdas pós-colheita de até 30\% dos frutos, quando provenientes de pomares com elevado risco e em safras agrícolas que propiciam a sua ocorrência (BASSO, 2002). O "bitter pit" caracteriza-se inicialmente como uma discreta mancha na polpa, de coloração escura, que se torna desidratada com o tempo, ocasionando pequenas depressões na epiderme do fruto (PESIS et al., 2009).

O baixo conteúdo de Ca nos frutos favorece a ocorrência de "bitter pit" em maçãs (SAURE, 2005; AMARANTE et al., 2011a). Além da deficiência de $\mathrm{Ca}$, o desenvolvimento de "bitter pit" está relacionado com elevados conteúdos de outros nutrientes nos frutos, principalmente $\mathrm{Mg}, \mathrm{K}$ e N (AMARANTE et al., 2006a e 2006b). Apesar de intensa pesquisa em busca de medidas de controle, atualmente, a pulverização do pomar com cloreto de cálcio $\left(\mathrm{CaCl}_{2}\right.$; 0,4-0,6\%) é o método mais utilizado para aumentar o teor de Ca no fruto e prevenir sua ocorrência. No entanto, em trabalho realizado com o objetivo de avaliar o efeito de diferentes números de pulverizações de $\mathrm{CaCl}_{2}$ na cultura da macieira, não foi constada redução na incidência de "bitter pit" e/ou aumento no teor de Ca nas folhas e frutos (ERNANI et al., 2008). Desta forma, faz-se necessário o estudo de outras medidas, visando a aumentar os teores de $\mathrm{Ca}$ e a reduzir a incidência de "bitter pit" em maçãs.

A pulverização de macieiras com giberelinas (GAs) tem sido utilizada para promover o crescimento dos frutos (LOONEY et al., 1992). Todavia, o excessivo crescimento dos frutos pode comprometer a funcionalidade do xilema e/ou diluir o $\mathrm{Ca}$ existente na polpa do fruto, com isso reduzindo o teor de $\mathrm{Ca}$ e aumentando as chances de ocorrência de "bitter pit” em maçãs (MIQUELOTO, 2011). Além disso, GAs ativas aumentam o crescimento vegetativo, ocasionando maior competição das folhas com os frutos, pelo Ca (BANGERTH, 1976), podendo assim reduzir os teores de Ca nos frutos e aumentar a suscetibilidade ao "bitter pit".

O composto prohexadiona-cálcio (ProCa) é um inibidor da biossíntese da GAs, impedindo oxidação da $\mathrm{GA}_{19}$ a $\mathrm{GA}_{20}$ ou GA 20 a GA, ou seja, não permite a conversão das GAs nas formas inativas a ativas, reduzindo assim o crescimento vegetativo (DAVIES, 2004). Portanto, o ProCa representa uma alternativa para reduzir o crescimento vegetativo em macieiras, diminuindo assim a competição entre folhas e frutos pelo $\mathrm{Ca}$, o que pode reduzir a ocorrência de "bitter pit" nos frutos.

Estudos recentes mostraram que o desenvolvimento do "bitter pit" nem sempre está relacionado ao conteúdo total de $\mathrm{Ca}$ encontrado no fruto. A homeostase anormal do Ca a nível celular, em que o esgotamento do Ca livre no apoplasto afeta a estrutura e a função da membrana plasmática, possivelmente está envolvida no desenvolvimento deste distúrbio fisiológico (FREITAS et al., 2010). O esgotamento do Ca livre no apoplasto pode ocorrer através do transporte deste mineral para o vacúolo, ficando complexados com fenóis, oxalatos e fosfatos (WHITE; BROADLEY, 2003), desta forma, não estando disponível para a preservação da integridade da membrana plasmática. O movimento do Ca para o vacúolo ocorre pela atividade da bomba Ca-ATPase, utilizando ATP como fonte de energia, e da proteína de transporte do tipo antiporte $\mathrm{Ca}^{2+} / \mathrm{H}^{+}(\mathrm{CAX})$, a qual utiliza o gradiente eletroquímico como fonte de energia para bombear o Ca para o interior desta organela (FREITAS et al., 2010). Além disto, também existem outras bombas de prótons, $\mathrm{H}^{+} /$pirofosfatase e ATPase vacuolar, que são responsáveis por gerar um gradiente eletroquímico no tonoplasto, com isso ativando as proteínas CAX e atuando de forma indireta no movimento do $\mathrm{Ca}$ para o interior do vacúolo (WHITE; BROADLEY, 2003). Portanto, a atividade destes transportadores é de grande importância para a homeostase do Ca a nível celular e para a manifestação do "bitter pit". Tem sido demonstrado, também, que o aumento nos níveis de giberelinas (GAs) pode estar relacionado com o desenvolvimento de distúrbios fisiológicos relacionados com a deficiência de Ca nos frutos (SAURE, 2005). Segundo Freitas e Mitcham (2010), a expressão dos genes das CAXs e Ca-ATPase em frutos de tomate aumenta pela atividade do hormônio $\mathrm{GA}_{3}$, com isso, podendo reduzir o nível de $\mathrm{Ca}$ apoplástico e aumetar a ocorrência de distúrbios fisiológicos ocasionados pela deficiência de $\mathrm{Ca}$.

Este trabalho foi desenvolvido com o objetivo de avaliar os efeitos da pulverização de macieiras com um inibidor da síntese de giberelinas, o prohexadiona-cálcio (ProCa), e com giberelina $\mathrm{GA}_{3}$, no crescimento vegetativo das plantas e na ocorrência de "bitter pit" em maçãs.

\section{MATERIAL E MÉTODOS}

O experimento foi conduzido em pomar da Empresa de Pesquisa Agropecuária e Extensão Rural de Santa Catarina S.A. (EPAGRI), na Estação 
Experimental de São Joaquim-SC (latitude $28^{\circ} 16^{\prime}$ $40,02^{\prime}$ ' S, longitude $49^{\circ} 56^{\prime} 09,10^{\prime}$ " W e altitude de $1.400 \mathrm{~m}$ ), na safra de 2009/2010. Foram utilizadas macieiras 'Catarina', com 14 anos de idade, sobre porta-enxerto 'Marubakaido', conduzidas com líder central e com espaçamento de $2 \mathrm{~m}$ entre plantas e $6,5 \mathrm{~m}$ entre filas. Também foram utilizadas macieiras 'Fuji', com 13 anos de idade, sobre porta-enxerto 'Marubakaido', com filtro M9, conduzidas com líder central, no espaçamento de 1,5 m entre plantas e 4,5 $\mathrm{m}$ entre filas. Esta área experimental não recebeu pulverizações com cloreto de cálcio $\left(\mathrm{CaCl}_{2}\right)$, visando a favorecer a ocorrência de "bitter pit".

As macieiras 'Catarina' e 'Fuji' foram pulverizadas com água (tratamento controle), prohexadionacálcio [ProCa; $319 \mathrm{mg}$ (i.a.) $\left.\mathrm{L}^{-1}\right]$ e giberelina $\left[\mathrm{GA}_{3}\right.$; $319 \mathrm{mg}$ (i.a.) $\left.\mathrm{L}^{-1}\right]$. As aplicações iniciaram na queda das pétalas (15-10-2009), quando as brotações do ano estavam com 5-10 cm de comprimento, sendo repetidas após 20 dias. Foi utilizado um volume de calda de $1.000 \mathrm{~L} \mathrm{ha}^{-1}$, procurando atingir o completo molhamento foliar de toda a planta. O delineamento experimental utilizado foi em blocos ao caso, segundo fatorial $3 \times 2$ (três tratamentos e duas cultivares), com cinco repetições, sendo cada repetição constituída de uma planta, totalizando 30 unidades amostrais.

No mês de janeiro, foram coletadas folhas (20 folhas/repetição), no terço médio dos ramos do ano, para as avaliações de teor de clorofila total, área foliar $\left(\mathrm{AF} ; \mathrm{cm}^{2}\right)$, massa seca $(\mathrm{g})$ e área foliar específica (AFE; $\mathrm{cm}^{2} \mathrm{~g}^{-1}$ ), segundo metodologia descrita por Amarante et al. (2011b).

Na maturação comercial, foi realizada a colheita dos frutos, que foram separados em amostras contendo 25 e 75 frutos por repetição, para as maçãs 'Catarina' e 'Fuji', respectivamente. Estes frutos foram armazenados em câmara fria convencional $\left(0 \pm 0,5{ }^{\circ} \mathrm{C} / 90-95 \%\right.$ UR $)$, durante quatro meses, seguido de cinco dias de comercialização simulada $\left(20 \pm 4^{\circ} \mathrm{C} / 60-70 \% \mathrm{UR}\right)$, e avaliados quanto à incidência (\%) e índice de "bitter pit". O índice de "bitter pit" foi determinado utilizando uma escala de 6 níveis de danos visuais na casca: nenhuma mancha (0); uma mancha (1); duas manchas (2); três manchas (3); quatro manchas (4); cinco manchas (5), e mais que cinco manchas (6) de "bitter pit". Para o cálculo do índice, foi utilizada a seguinte fórmula, decrita por Pesis et al. (2009):
Para a análise mineral dos frutos, estes foram previamente separados em amostras contendo 20 frutos por repetição e em seguida lavados com água destilada. Posteriormente, os frutos foram cortados na região equatorial, sendo utilizada apenas a parte distal, retirando amostras do tecido da casca. Foi utilizado apenas o tecido da casca, pois o mesmo é mais indicado para a quantificação dos teores minerais, visando a discriminar maçãs quanto à ocorrência de "bitter pit" (AMARANTE et al., 2006a, 2006b e 2011a). O tecido da casca foi processado, conforme a metodologia descrita por Miqueloto (2011). Para a determinação do teor de $\mathrm{Ca}$, retirou-se uma alíquota de $5 \mathrm{~mL}$ do extrato original e adicionaram-se $5 \mathrm{~mL}$ de óxido de lantânio, efetuando a leitura do teor mineral no equipamento de absorção atômica (modelo A analyst 100). Para a determinação de $\mathrm{Mg}$, retiraramse $2 \mathrm{~mL}$ do extrato original e adicionaram-se $10 \mathrm{~mL}$ de água destilada. Desta solução, pipetaram-se $5 \mathrm{~mL}$ e procedeu-se à metodologia descrita para a determinação de $\mathrm{Ca}$. Para a determinação de $\mathrm{K}$, retirou-se $1 \mathrm{~mL}$ do extrato original e adicionaram-se $7 \mathrm{~mL}$ de água destilada, e realizaram-se a leitura em fotômetro de chama (Digimed DM-61). O N foi determinado pelo método semimicro Kjeldahl.

No mês de maio de 2010, foram realizadas as avaliações de comprimento dos ramos do ano, localizados no terço médio da planta, com uma fita métrica, e pesagem dos ramos podados, com uma balança analógica.

Os dados coletados foram analisados utilizando o programa estatístico SAS (2002). As médias de tratamentos foram comparadas pelo teste de Tukey $(\mathrm{p}<0,05)$.

Índice de "bitter pit" $=\sum_{0}^{6} \frac{\text { (nível do índice) } x\left(n^{\circ} \text { frutos a este nível) }\right.}{\left(\text { no total de frutos) }^{\circ}\right.}$ 


\section{RESULTADOS E DISCUSSÃO}

Houve efeito de tratamentos e cultivares, e interação significativa $(\mathrm{p}<0,05)$ entre estes fatores, para os atributos foliares e de crescimento vegetativo das plantas (Tabela 1).

O tratamento com ProCa apresentou menor AF, porém, apenas em 'Catarina', esta diferença foi significativa em relação ao controle (Tabela 1). Os maiores valores de AF foram verificados no tratamento com $\mathrm{GA}_{3}$, mas esta diferença foi significativa em relação ao controle apenas em 'Fuji'. Na média dos valores das duas cultivares, o tratamento com ProCa apresentou AF significativamente menor do que o controle, ao passo que o tratamento com $\mathrm{GA}_{3}$ apresentou maior AF em relação ao controle (Tabela 1).

A AFE do tratamento com ProCa foi menor em relação ao controle em 'Fuji', sendo que, para o tratamento com $\mathrm{GA}_{3}$, a AFE foi maior em relação ao controle em 'Catarina' (Tabela 1). Na média dos valores das duas cultivares, apenas o tratamento com ProCa apresentou menor AFE em relação ao controle, mostrando que a inibição da síntese de GAs resulta em folhas menores e com mésofilo foliar mais espesso.

Apenas em 'Catarina', o teor de clorofila foliar foi menor no tratamento com $\mathrm{GA}_{3}$, em relação aos tratamentos-controle e ProCa (Tabela 1). O mesmo ocorre ao compararmos a média dos valores das duas cultivares. Este comportamento pode ser explicado pela maior área foliar em macieiras tratadas com $\mathrm{GA}_{3}$ (Tabela 1), o que resulta em redução na espessura em detrimento ao incremento na área das folhas. Com a redução da espessura do mesófilo das folhas, há menor acúmulo de clorofila por unidade de área.

Em ambas as cultivares, o comprimento médio dos ramos do ano foi significativamente menor no tratamento com ProCa, e maior no tratamento com $\mathrm{GA}_{3}$, comparativamente ao controle (Tabela 1). Considerando os valores médios das duas cultivares, o ProCa reduziu em 56\%, e o $\mathrm{GA}_{3}$ aumentou em $22 \%$ o comprimento médio dos ramos, em relação ao controle (Tabela 1). Os valores de peso dos ramos na poda foram menores no tratamento com ProCa, e maiores no tratamento com $\mathrm{GA}_{3}$, em ambas as cultivares. Porém, apenas em 'Fuji' houve diferença significativa em relação ao controle do tratamento com GA. No entanto, na média dos valores das duas cultivares, o peso dos ramos na poda foi significativamente menor no tratamento com ProCa e maior no tratamento com $\mathrm{GA}_{3}$, comparativamente ao controle. Neste caso, o ProCa reduziu em 33\% e o $\mathrm{GA}_{3}$ aumentou em $32 \%$ o peso dos ramos na poda, em relação ao controle.

Estes resultados estão de acordo trabalhos anteriores, mostrando o efeito do ProCa sobre a redução na área foliar (GUAK et al., 2001; MEDJDOUB, 2003), no comprimento dos ramos (COSTA et al., 2004; BASAK, 2004) e no peso de ramos podados (PETRI; LEITE, 2005) em macieiras. Este comportamento está relacionado ao efeito do ProCa, que bloqueia a ação das enzimas dioxigenases $\left(\mathrm{GA}_{20}\right.$-oxidase e $\mathrm{GA}_{3}$-oxidase), impedindo a formação de GAs ativas (DAVIES, 2004). Portanto, esta redução no crescimento vegetativo, tanto em área foliar quanto no comprimento médio dos ramos do ano e no peso dos ramos na poda, deve-se ao efeito do ProCa na inibição da elongação e divisão celular (MEDJDOUB, 2003).

A redução no crescimento vegetativo em macieiras tratadas com o ProCa (Tabela 1) pode apresentar alguns benefícios ao manejo das plantas, tais como a maior eficiência na pulverização de produtos químicos, especialmente $\mathrm{CaCl}_{2}$, utilizado para aumentar o teor de $\mathrm{Ca}$ nos frutos e, assim, reduzir a ocorrência de distúrbios fisiológicos pós-colheita. Além disso, também pode contribuir para a redução no tempo e no custo para a realização da poda.

Em ambas as cultivares, a incidência de "bitter pit" (\%) após o período de armazenamento foi significativamente menor no tratamento com ProCa, e maior no tratamento com $\mathrm{GA}_{3}$, comparativamente ao controle (Tabela 2). O mesmo correu para o índice de "bitter pit" em 'Catarina', sendo que em 'Fuji' este índice foi menor no tratamento com ProCa, porém não houve diferença entre os tratamentos-controle e $\mathrm{GA}_{3}$. Considerando os valores médios das duas cultivares, o tratamento com ProCa reduziu em 14\% a incidência e em 40\% o índice de "bitter pit", em relação ao tratamento-controle.

Medjdoub (2003) observou redução na incidência de "bitter pit", após o período de armazenamento, em frutos de macieira 'Golden Smoothee' tratadas com ProCa. Segundo este autor, em geral, a aplicação de inibidores de crescimento, como o daminozide e paclobutrazol, resulta em menor incidência de "bitter pit" e outros distúrbios fisiológicos.

Não houve diferença entre o tratamento-controle e os tratamentos com ProCa e $\mathrm{GA}_{3}$ quanto aos teores de $\mathrm{Ca}$ e as relações $\mathrm{N} / \mathrm{Ca}$ e $(\mathrm{K}+\mathrm{Mg}+\mathrm{N}) / \mathrm{Ca}$ na casca de maçãs 'Catarina', e a relação $\mathrm{Mg} / \mathrm{Ca}$ na casca de maçãs 'Fuji' (Tabela 3). O tratamento com ProCa reduziu a relação $\mathrm{K} / \mathrm{Ca}$ em 'Catarina' e a relação $\mathrm{N} / \mathrm{Ca}$ 
em 'Fuji', em relação ao controle. O tratamento com $\mathrm{GA}_{3}$ reduziu o teor de $\mathrm{Ca}$ e aumentou as relações $\mathrm{K} /$ $\mathrm{Ca}$ e $(\mathrm{K}+\mathrm{Mg}+\mathrm{N}) / \mathrm{Ca}$ na casca em 'Fuji', e aumentou a relação $\mathrm{Mg} / \mathrm{Ca}$ na casca em 'Catarina'.

Ao considerarmos os valores médios dos atributos minerais das duas cultivares, as relações $\mathrm{N} / \mathrm{Ca}$ e $(\mathrm{K}+\mathrm{Mg}+\mathrm{N}) / \mathrm{Ca}$ na casca foram significativamente menores no tratamento com ProCa do que no controle, ao passo que o tratamento com $\mathrm{GA}_{3}$ apresentou menores teores de $\mathrm{Ca}$ e maiores valores das relações $\mathrm{K} / \mathrm{Ca}, \mathrm{Mg} / \mathrm{Ca}$ e $(\mathrm{K}+\mathrm{Mg}+\mathrm{N}) / \mathrm{Ca}$ na casca em relação ao controle (Tabela 3 ).

$\mathrm{O}$ tratamento com ProCa reduziu a incidência e o índice de "bitter pit" nos frutos (Tabela 2) devido a um melhor equilíbrio na composição mineral, associado aos menores valores das relações N/Ca e $(\mathrm{K}+\mathrm{Mg}+\mathrm{N}) / \mathrm{Ca}$ no tecido da casca (Tabela 3$)$. Já os maiores valores de incidência e índice de "bitter pit" nos frutos do tratamento com $\mathrm{GA}_{3}$ (Tabela 2) parecem estar associados aos menores teores de $\mathrm{Ca}$ e maiores valores das relações $\mathrm{K} / \mathrm{Ca}, \mathrm{Mg} / \mathrm{Ca}$ e $(\mathrm{K}+\mathrm{Mg}+\mathrm{N}) /$ $\mathrm{Ca}$ na casca dos frutos deste tratamento (Tabela 3). Possivelmente, estes resultados estão relacionados ao maior crescimento vegetativo proporcionado pelo tratamento com $\mathrm{GA}_{3}$ (Figura 1), o que possibilitou o aumento na competição do Ca disponível entre frutos e ramos (folhas e brotos) (BANGERTH, 1976). Além disso, em condições de elevados níveis endógenos de GAs e baixos níveis de auxinas, existe o favorecimento na formação de floema, o qual corresponde ao tecido vascular responsável pela translocação dos nutrientes $\mathrm{N}, \mathrm{K}$ e Mg para os frutos, especialmente no final da estação de crescimento (SAURE, 2005). Portanto, nestas condições, continua ocorrendo a translocação de $\mathrm{K}, \mathrm{N}$ e $\mathrm{Mg}$ para os frutos, podendo resultar em maior acúmulo destes nutrientes minerais em relação ao $\mathrm{Ca}$, tendo em vista que o $\mathrm{Ca}$ é praticamente imóvel no floema, levando à manifestação do "bitter pit" (SAURE, 2005).

A menor ocorrência de "bitter pit" nos frutos do tratamento com ProCa pode estar associada, além dos menores valores das relações $\mathrm{N} / \mathrm{Ca}$ e $(\mathrm{K}+\mathrm{Mg}+\mathrm{N}) /$ Ca no tecido da casca, aos efeitos na homeostase celular de Ca. Segundo Freitas e Mitcham (2010), tomates tratados com ProCa apresentaram aumento no conteúdo de $\mathrm{Ca}$ apoplástico nos tecidos do pericarpo, com isso resultando em frutos sem podridão estilar, distúrbio que é causado pela deficiência de $\mathrm{Ca}$. Segundo os mesmos autores, esta resposta se deve à redução na expressão dos genes das CAXs e Ca-ATPase nos frutos de tomates tratados com Pro$\mathrm{Ca}$. Portanto, isto reduz o aprisionamento do $\mathrm{Ca}$ no interior do vacúolo, ficando disponível no apoplasto e para a membrana plasmática, evitando assim a manifestação do distúrbio fisiológico. Mecanismo similar pode também explicar a menor incidência e o índice de "bitter pit" em maçãs colhidas de plantas tratadas com ProCa (Tabela 2), já que os teores de Ca na casca dos frutos não diferiram entre os tratamentos ProCa e controle (Tabelas 3).

$\mathrm{O}$ tratamento com ProCa aumentou o teor Ca das folhas apenas em macieiras 'Fuji' (dados não apresentados). O mesmo foi observado em macieiras 'Fuji', 'Royal Gala'e 'Golden Smoothee' tratadas com ProCa (MEDJDOUB, 2003). Este comportamento pode estar relacionado à menor área foliar específica e/ou ao menor crescimento dos ramos do ano (Tabela 1) em plantas tratadas com ProCa, com isso resultando em maior teor de Ca nas folhas. No entanto, não houve diferença quanto aos teores de $\mathrm{K}$, $\mathrm{Mg}$ e $\mathrm{N}$ nas folhas entre os tratamentos, em ambas as cultivares (dados não apresentados).

A redução na incidência e no índice de "bitter pit" nos frutos em macieiras tratadas com ProCa mostra que este produto pode ser uma nova alternativa para o controle deste distúrbio fisiológico em pomares e/ou em anos com elevado risco de sua ocorrência. Isto possibilita a redução nas perdas pós-colheita causadas pelo "bitter pit", bem como reduz custos de mão de obra com operações de poda e manejo das plantas, tendo em vista seu menor crescimento vegetativo. 
TABELA 1 - Área, área específica e teor de clorofila nas folhas, comprimento médio dos ramos do ano e peso dos ramos removidos na poda de inverno, em macieiras 'Catarina' e 'Fuji' submetidas aos tratamentos-controle, com prohexadiona-cálcio (ProCa) e giberelina $\left(\mathrm{GA}_{3}\right)$.

\begin{tabular}{|c|c|c|c|}
\hline Tratamentos & 'Catarina' & 'Fuji' & Média \\
\hline & \multicolumn{3}{|c|}{ Área foliar $\left(\mathrm{cm}^{2}\right)$} \\
\hline Controle & $28,5 \mathrm{aA}$ & $29,5 \mathrm{bA}$ & $29,0 \mathrm{~b}$ \\
\hline ProCa & $23,7 \mathrm{bA}$ & $26,5 \mathrm{bA}$ & $25,1 \mathrm{c}$ \\
\hline $\mathrm{GA}_{3}$ & $31,7 \mathrm{aA}$ & 33,9 aA & $32,8 \mathrm{a}$ \\
\hline Média & $27,9 \mathrm{~B}$ & $29,9 \mathrm{~A}$ & \\
\hline \multirow[t]{2}{*}{$\mathrm{CV}(\%)$} & 14,1 & 12,2 & \\
\hline & \multicolumn{3}{|c|}{ Área foliar específica $\left(\mathrm{cm}^{2} \mathrm{~g}^{-1}\right)$} \\
\hline Controle & $6,1 \mathrm{bB}$ & $7,4 \mathrm{aA}$ & $6,7 \mathrm{a}$ \\
\hline ProCa & $5,3 \mathrm{bA}$ & $6,0 \mathrm{bA}$ & $5,7 \mathrm{~b}$ \\
\hline $\mathrm{GA}_{3}$ & $7,1 \mathrm{aA}$ & $6,8 \mathrm{abA}$ & $7,0 \mathrm{a}$ \\
\hline Média & $6,2 \mathrm{~B}$ & $6,7 \mathrm{~A}$ & \\
\hline \multirow[t]{2}{*}{ CV $(\%)$} & 13,9 & 11,5 & \\
\hline & \multicolumn{3}{|c|}{ Teor foliar de clorofila (unidades SPAD) } \\
\hline Controle & $63,7 \mathrm{aA}$ & $49,8 \mathrm{aB}$ & $56,7 \mathrm{a}$ \\
\hline ProCa & 56,6 aA & $52,0 \mathrm{aA}$ & $54,3 \mathrm{a}$ \\
\hline $\mathrm{GA}_{3}$ & $44,6 \mathrm{bA}$ & $45,7 \mathrm{aA}$ & $45,1 \mathrm{~b}$ \\
\hline Média & $55,0 \mathrm{~A}$ & $49,1 \mathrm{~B}$ & \\
\hline \multirow[t]{2}{*}{$\mathrm{CV}(\%)$} & 19,7 & 5,9 & \\
\hline & \multicolumn{3}{|c|}{ Comprimento médio dos ramos do ano $(\mathrm{cm})$} \\
\hline Controle & $55,4 \mathrm{bA}$ & $51,5 \mathrm{bA}$ & $53,5 \mathrm{~b}$ \\
\hline ProCa & $25,4 \mathrm{cA}$ & $21,9 \mathrm{cA}$ & $23,7 \mathrm{c}$ \\
\hline $\mathrm{GA}_{3}$ & $69,4 \mathrm{aA}$ & $68,6 \mathrm{aA}$ & $69,0 \mathrm{a}$ \\
\hline Média & $50,1 \mathrm{~A}$ & 47,4 A & \\
\hline \multirow[t]{2}{*}{ CV $(\%)$} & 34,2 & 45,6 & \\
\hline & \multicolumn{3}{|c|}{ Peso dos ramos da poda (kg/planta) } \\
\hline Controle & $3,5 \mathrm{abA}$ & 3,8 bA & $3,6 \mathrm{~b}$ \\
\hline ProCa & $2,0 \mathrm{bA}$ & $2,7 \mathrm{bA}$ & $2,4 \mathrm{c}$ \\
\hline $\mathrm{GA}_{3}$ & $4,3 \mathrm{aB}$ & $6,3 \mathrm{aA}$ & $5,3 \mathrm{a}$ \\
\hline Média & $3,3 \mathrm{~B}$ & $4,3 \mathrm{~A}$ & \\
\hline CV $(\%)$ & 49,5 & 50,1 & \\
\hline
\end{tabular}

Médias seguidas de mesma letra, minúsculas nas colunas e maiúsculas nas linhas, não diferem entre si, pelo teste de Tukey ( $\mathrm{p}<0,05$ ).

TABELA 2 - Incidência e índice de "bitter pit" nos frutos após o período de armazenamento, em macieiras 'Catarina' e 'Fuji' submetidas aos tratamentos-controle, com prohexadiona-cálcio (ProCa) e giberelina $\left(\mathrm{GA}_{3}\right)$.

\begin{tabular}{lccc}
\hline Tratamentos & 'Catarina' & 'Fuji' & Média \\
\hline & & Incidência de "bitter pit" (\%) & \\
Controle & $62,3 \mathrm{bA}$ & $63,0 \mathrm{bA}$ & $62,6 \mathrm{~b}$ \\
ProCa & $52,1 \mathrm{cA}$ & $55,4 \mathrm{cA}$ & $53,7 \mathrm{c}$ \\
$\mathrm{GA}_{3}$ & $77,4 \mathrm{aA}$ & $77,2 \mathrm{aA}$ & $77,3 \mathrm{a}$ \\
\hline Média & $63,9 \mathrm{~A}$ & $65,2 \mathrm{~A}$ \\
$\mathrm{CV}(\%)$ & 11,5 & 9,0 & \\
\hline & & Índice de "bitter pit" (0-6) & \\
Controle & $1,9 \mathrm{bA}$ & $2,1 \mathrm{aA}$ & $2,0 \mathrm{~b}$ \\
ProCa & $1,2 \mathrm{cA}$ & $1,1 \mathrm{bA}$ & $1,2 \mathrm{c}$ \\
$\mathrm{GA}$ & $2,8 \mathrm{aA}$ & $2,3 \mathrm{aA}$ & $2,6 \mathrm{a}$ \\
\hline Média & $2,0 \mathrm{~A}$ & $1,9 \mathrm{~A}$ & \\
$\mathrm{CV}(\%)$ & 18,8 & 23,3 & \\
\hline
\end{tabular}

Médias seguidas de mesma letra, minúsculas nas colunas e maiúsculas nas linhas, não diferem entre si, pelo teste de Tukey $(\mathrm{p}<0,05)$. 
TABELA 3 - Teores de $\mathrm{Ca}$ (mg/kg de massa fresca) e valores das relações $\mathrm{K} / \mathrm{Ca}, \mathrm{Mg} / \mathrm{Ca}, \mathrm{N} / \mathrm{Ca}$ e (K+Mg+N)/ Ca no tecido da casca dos frutos, em macieiras 'Catarina' e 'Fuji' submetidas aos tratamentoscontrole, com prohexadiona-cálcio (ProCa) e giberelina $\left(\mathrm{GA}_{3}\right)$.

\begin{tabular}{|c|c|c|c|}
\hline Tratamentos & 'Catarina' & 'Fuji' & Média \\
\hline & & $\mathrm{Ca}$ & \\
\hline Controle & $155 \mathrm{abA}$ & $139 \mathrm{aB}$ & $147 \mathrm{a}$ \\
\hline ProCa & $172 \mathrm{aA}$ & $138 \mathrm{aB}$ & $155 \mathrm{a}$ \\
\hline $\mathrm{GA}_{3}$ & $139 \mathrm{bA}$ & $110 \mathrm{bB}$ & $125 \mathrm{~b}$ \\
\hline Média & $155 \mathrm{~A}$ & $129 \mathrm{~B}$ & \\
\hline \multirow[t]{2}{*}{ CV $(\%)$} & 24,8 & 20,2 & \\
\hline & & $\mathrm{K} / \mathrm{Ca}$ & \\
\hline Controle & $11,0 \mathrm{aA}$ & $7,4 \mathrm{bB}$ & $9,2 \mathrm{~b}$ \\
\hline ProCa & $8,3 \mathrm{bA}$ & $8,3 \mathrm{bA}$ & $8,3 \mathrm{~b}$ \\
\hline $\mathrm{GA}_{3}$ & $11,1 \mathrm{aA}$ & $11,5 \mathrm{aA}$ & $11,3 \mathrm{a}$ \\
\hline Média & $10,1 \mathrm{~A}$ & $9,1 \mathrm{~A}$ & \\
\hline \multirow[t]{2}{*}{ CV $(\%)$} & 37,6 & 35,8 & \\
\hline & & $\mathrm{Mg} / \mathrm{Ca}$ & \\
\hline Controle & $1,10 \mathrm{bB}$ & $1,30 \mathrm{abA}$ & $1,20 \mathrm{~b}$ \\
\hline ProCa & $0,93 \mathrm{bB}$ & $1,16 \mathrm{bA}$ & $1,05 \mathrm{~b}$ \\
\hline $\mathrm{GA}_{3}$ & $1,37 \mathrm{aB}$ & 1,46 aA & $1,42 \mathrm{a}$ \\
\hline Média & $1,13 \mathrm{~B}$ & $1,31 \mathrm{~A}$ & \\
\hline \multirow[t]{2}{*}{ CV $(\%)$} & 26,9 & 35,5 & \\
\hline & & $\mathrm{N} / \mathrm{Ca}$ & \\
\hline Controle & $6,8 \mathrm{aA}$ & $6,0 \mathrm{aA}$ & $6,4 \mathrm{a}$ \\
\hline ProCa & $6,3 \mathrm{aA}$ & $3,7 \mathrm{bB}$ & $5,0 \mathrm{~b}$ \\
\hline $\mathrm{GA}_{3}$ & $6,6 \mathrm{aB}$ & 7,7 aA & $7,1 \mathrm{a}$ \\
\hline Média & $6,6 \mathrm{~A}$ & $5,8 \mathrm{~A}$ & \\
\hline \multirow[t]{2}{*}{ CV (\%) } & 39,7 & 39,9 & \\
\hline & & $+\mathrm{Mg}+\mathrm{N}) / \mathrm{Ca}$ & \\
\hline Controle & $18,9 \mathrm{abA}$ & $15,4 \mathrm{bA}$ & $17,1 \mathrm{~b}$ \\
\hline ProCa & $15,6 \mathrm{bA}$ & $13,2 \mathrm{bA}$ & $14,4 \mathrm{c}$ \\
\hline $\mathrm{GA}_{3}$ & 19,8 aA & $20,7 \mathrm{aA}$ & $20,2 \mathrm{a}$ \\
\hline Média & $18,1 \mathrm{~A}$ & $16,4 \mathrm{~A}$ & \\
\hline CV (\%) & 31,7 & 32,6 & \\
\hline
\end{tabular}

Médias seguidas de mesma letra, minúsculas nas colunas e maiúsculas nas linhas, não diferem entre si, pelo teste de Tukey ( $\mathrm{p}<0,05$ ).

\section{CONCLUSÕES}

Em macieiras 'Catarina' e 'Fuji', o crescimento vegetativo é significativamente menor nas plantas tratadas com ProCa, e maior naquelas tratadas com $\mathrm{GA}_{3}$, comparativamente ao controle. Maçãs 'Catarina' e 'Fuji' tratadas com ProCa tem menor ocorrência de "bitter pit" após o período de armazenamento, associada aos menores teores de $\mathrm{K}$, $\mathrm{Mg}$ e $\mathrm{N}$, em relação aos teores de $\mathrm{Ca}$, no tecido da casca, comparativamente ao tratamento-controle. Já o tratamento com $\mathrm{GA}_{3}$ aumenta a ocorrência de "bitter pit" em relação ao controle, em ambas as cultivares.

\section{AGRADECIMENTOS}

Os autores agradecem ao CNPq e à CAPES, pela concessão de bolsas, à FAPESC, pelo apoio fi- nanceiro, e à EPAGRI de São Joaquim-SC, por ceder a área experimental para a execução deste projeto.

\section{REFERÊNCIAS}

AMARANTE, C.V.T.; CHAVES, D.V.; ERNANI, P.R. Análise multivariada de atributos nutricionais associados ao "bitter pit" em maçãs 'Gala'. Pesquisa Agropecuária Brasileira, Brasília, v.41, n.5, p.841$846,2006 a$.

AMARANTE, C.V.T.; CHAVES, D.V.; ERNANI, P.R. Composição mineral e severidade de "bitter pit” em maçãs 'Catarina'. Revista Brasileira de Fruticultura, Jaboticabal, v.28, n.1, p.51-54, 2006b. 
AMARANTE, C.V.T.; ERNANI, P.R.; STEFFENS, C.A.; ARGENTA, L.C. O teor de cálcio na casca é indicativo da suscetibilidade ao "bitter pit" em maçãs 'Fuji'. Revista Brasileira de Fruticultura, Jaboticabal, v.33, n.1, p.180-186, 2011a.

AMARANTE, C.V.T.; STEFFENS, C.A.; ARGENTA, L.C. Yield and fruit quality of 'Gala' and 'Fuji' apple trees protected by white anti-hail net. Scientia Horticulturae, Amsterdam, v.129, n.1, p.79-85, $2011 b$.

BANGERTH, F. A role for auxin and auxin transport inhibitors on the $\mathrm{Ca}$ content of artificially induced parthenocarpic fruits. Physiologia Plantarum, Copenhagen, v.37, n.3, p.191-194, 1976.

BASAK, A. Growth and fruiting of 'Elstar' apple trees in response to prohexadione calcium depending on the rootstock. Acta Horticulturae, The Hague, n.653, p.117-126, 2004.

BASSO, C. A cultura da macieira: distúrbios fisiológicos. 2.ed. Florianópolis: Epagri, 2002. p.609-636.

COSTA, G.; SABATINI, F.; SPINELLI, F.; ANDREOTTI, C.; BOMBEN, C.; VIZZOTO, G. Two years of application of prohexadione-Ca on apple: effect on vegetative and cropping performance, fruit quality, return bloom and residual effect. Acta Horticulturae, The Hague, n.653, p.27-132, 2004.

DAVIES, P.J. Plant hormones: biosynthesis, signal transduction and action. 3. ed. Dordrecht: Netherlands, 2004. 775p.

ERNANI, P.R.; DIAS, J.; AMARANTE, C.V.T.; RIBEIRO, D.C.; ROGERI, D.A. Preharvest calcium sprays were not always needed to improve fruit quality of 'Gala' apples in Brazil. Revista Brasileira de Fruticultura, Jaboticabal, v.30, n.4, p.892-896, 2008.

FREITAS, S.T.; AMARANTE, C.V.T.; LABAVITCH, J.M.; MITCHAM, E.J. Cellular approach to understand bitter pit development in apple fruit. Postharvest Biology and Technology, Amsterdam, v.57, n.1, p.6-13, 2010.

FREITAS, S.T.; MITCHAM, E.J. Effects of growth regulators on calcium deficiency disorders. In: MEETING WESTERN PLANT GROWTH REGUlATOR SOCIETY, 22., 2010, Davis. Abstracts... Disponível em: <http://www.wpgrs.
org/2010WPGRSAbstract_Freitas.pdf $>$. Acesso em: 15 nov. 2010.

GUAK, S.; NEILSEN, D.; LOONEY, N.E. Growth, allocation of $\mathrm{N}$ and carbohydrates, and stomatal conductance of greenhouse grown apple treated with prohexadione-Ca and gibberellins. Journal of Horticultural Science \& Biotechnology, Ashford, v.76, n.6, p.746-752, 2001.

LOONEY, N.E.; GRANGER R.L.; CHU, C.L.; MANDER, L.N.; PHARIS, R.P. Influences of gibberellins $\mathrm{A}_{4}, \mathrm{~A}_{4+7}$ and $\mathrm{A}_{4+\text { iso-A } 7}$ on apple fruit quality and tree productivity. II. Other effects on fruit quality and importance of fruit position within the tree canopy. Journal of Horticultural Science \& Biotechnology, Ashford, v.67, n.6, p.841-847, 1992.

MEDJDOUB, R. Evaluación del prohexadioneCa para el control del crecimiento en manzano: efectos agronómicos y fisiológicos. 2003. 247 f. Tesis (Doctoral) - Universitat de Lleida, Lleida, 2003.

MIQUELOTO, A. Atributos minerais e aspectos fisiológicos relacionados com a ocorrência de “bitter pit” em maçãs. 2011. 56 f. Dissertação (Mestrado) - Universidade do Estado de Santa Catarina, Lages, 2011.

PESIS, E.; IBÁÑEZ, A.M.; PHU, M.L.; MITCHAM, E.J.; EBELER, S.E.; DANDEKAR, A.M. Superficial scald and bitter pit development in cold-stored transgenic apples suppressed for ethylene biosynthesis. Journal of Agricultural and Food Chemistry, Columbus, v.57, n.7, p.2786-2792, 2009.

PETRI, J.L.; LEITE, G.B. Control of 'Gala' and 'Fuji' apple tree growth by prohexadione-Ca. In: INTERNATIONAL SYMPOSIUM PLANT BIOREGULATORS IN FRUIT PRODUCTION, 10., 2005, Saltillo. Abstracts... p.41.

SAS INSTITUTE. Getting started with the SAS learning edition. Cary, 2002. 200p.

SAURE, M.C. Calcium translocation to fleshy fruit: its mechanism and endogenous control. Scientia Horticulturae, Amsterdam, v.105, n.1, p.65-89, 2005.

WHITE, P.J.; BROADLEY, M.R. Calcium in plants. Annals of Botany, London, v.92, n.4, p.487-511, 2003. 